\title{
ESTIMACIÓN DE LA INCERTIDUMBRE EN LA CALIBRACIÓN DE UN MATRAZ
}

\author{
María Lorena Blanco Rojas \\ Paulina Silva Trejos
}

\begin{abstract}
Resumen
Se presenta el desarrollo de un método para la estimación de la incertidumbre aplicado a un matraz volumétrico de $100 \mathrm{~cm}^{3}$, calibrado utilizando el principio gravimétrico.

Los resultados de la calibración fueron obtenidos de la participación en la ronda costarricense de volumen denominada Comparación interlaboratorial en volumen ENA-MET-001, organizada por el Laboratorio Costarricense de Metrología (LACOMET) que consistió en la calibración de un matraz de vidrio de $100 \mathrm{~cm}^{3}$ utilizando el método gravimétrico y la estimación de la incertidumbre. En el ejercicio participaron catorce laboratorios de ensayo y tres laboratorios de calibración; las autoras participaron desde dos laboratorios de ensayo de la Universidad de Costa Rica, obteniendo participaciones satisfactorias.

La estimación de la incertidumbre se realizó para informar los resultados de la intercomparación y se consideró importante su divulgación a fin de que pueda ser utilizada de ejemplo para laboratorios de ensayo y de calibración y también para fines didácticos en la preparación de personal técnico en metrología.
\end{abstract}

Palabras claves: volumen, metrología, incertidumbre, método, calibración, laboratorio, matraz.

\begin{abstract}
A method for estimation of uncertainty in volume measurement of a $100 \mathrm{~cm}^{3}$ flask calibrated by the gravimetric method is presented. The results of the calibration were obtained from the participation in the Costa Rican volume comparison round labeled: Interlaboratory volume comparison ENA-MET-001 organized by LACOMET (Laboratorio Costarricense de Metrología) that consisted of the calibration of a $100 \mathrm{~cm}^{3}$ flask using the gravimetric method and the estimation of uncertainty. Fourteen testing laboratories and three calibration laboratories participated in this exercise. The authors participated in two of the testing laboratories of the University of Costa Rica obtaining satisfactory results.

The estimation of uncertainty was done in order to communicate the results of the inter comparison round. These results are considered very important for testing and calibration laboratories and for teaching purposes to prepare technical personnel in Metrology.
\end{abstract}

Keywords: volume, metrology, uncertainty, method, calibration, laboratory, glass, volumetric.

Recibido: 17 de setiembre del 2009 • Aprobado: 17 de noviembre del 2009

\section{INTRODUCCIÓN}

Las mediciones volumétricas son esenciales para muchos de los ensayos que se practican rutinariamente en los laboratorios de ensayo; en estos laboratorios muchas veces se debe verificar la cristalería, por lo que es importante contar con métodos de estimación de la incertidumbre del resultado.
En los laboratorios, el uso de cristalería como matraces y pipetas es muy común, se requieren para preparar disoluciones patrón. En la mayoría de los casos, la contribución a la incertidumbre de esta etapa del ensayo es variable de influencia en el cálculo de la incertidumbre del resultado final de la medición, por tanto, se debe demostrar su trazabilidad, lo que hace obligatorio calibrar 
la cristalería, o bien, comprar cristalería calibrada acompañada de su certificado.

Según Sánchez, Picado, Sánchez y Sequeira (2001) "los equipos volumétricos utilizados en los análisis afectan los resultados de áreas tan importantes como la salud, el ambiente y la investigación, de ahí que es necesario garantizar los niveles de exactitud de estos equipos". Por razones económicas, es inusual que un laboratorio de ensayo envíe a calibrar toda la cristalería, la práctica es enviar a calibrar un instrumento por clase, y, verificar internamente el resto de la cristalería utilizando como patrones internos de referencia la cristalería calibrada externamente por laboratorios de calibración acreditados.

El equipo volumétrico utilizado corrientemente incluye pipetas, micropipetas, matraces o balones y probetas. La cristalería se clasifica según la tolerancia o máximo error permitido, en Clase A, los de mayor exactitud y en Clase B, los de menor exactitud, los de clase A son los utilizados en los laboratorios de ensayo. La capacidad de estas medidas varía desde $0,1 \mathrm{~cm}^{3}$ hasta $2000 \mathrm{~cm}^{3}$ y el error máximo permisible depende del tipo, la capacidad y la clase de exactitud.

En el Cuadro 1 se presentan los requerimientos para matraces tomados de la norma ASTM E 1878-97 (ASTM, 2009c).

Para los matraces aforados, la capacidad de un matraz se define como el volumen de agua a $20{ }^{\circ} \mathrm{C}$ expresado en centímetros cúbicos, contenida o vertida por el matraz a $20{ }^{\circ} \mathrm{C}$ con respecto a su trazo. El cuerpo del matraz tiene forma semejante a una pera con una base en el fondo sobre la cual el matraz puede mantenerse en posición vertical, cuando se coloca en una superficie horizontal. La boca de un matraz puede ser o no esmerilada y tener diferentes formas. Estos instrumentos son utilizados generalmente para contener volúmenes en la preparación de disoluciones patrón. El método de ajuste del menisco más comúnmente utilizado para disoluciones transparentes, consiste en que el punto más bajo del menisco coincida tangencialmente con la línea de graduación del instrumento de acuerdo con el método ASTM E 694-99 (ASTM, 2009d). La capacidad de un matraz no debe diferir de su capacidad nominal por un valor mayor que el error máximo permisible informado en el Cuadro 2 y tomado del método ASTM E 288-06 (ASTM, 2009a).

Existen diversos factores que afectan la exactitud de la medición del volumen para instrumentos de vidrio, entre ellos se pueden citar los siguientes.

Temperatura del recipiente: La medida da valores correctos del volumen de líquido medido para la temperatura de referencia a la cual se determina su capacidad durante la calibración inicial. El coeficiente cúbico de expansión térmica del vidrio del cual se fabrican los instrumentos volumétricos, está comprendido aproximadamente en el intervalo entre $\left(10 \cdot 10^{-6}\right.$ y $\left.25 \cdot 10^{-6}\right){ }^{\circ} \mathrm{C}^{-1}$. Como medida de seguridad se

Cuadro 1. Requerimientos para los matraces.

$\begin{array}{cccc}\text { Tipo } & \text { Capacidad } & \text { Tolerancia } & \begin{array}{c}\text { Diámetro interno } \\ \text { al aforo } \\ \left(\mathrm{cm}^{3}\right)\end{array} \\ 1 & 100 & \left(\mathrm{~cm}^{3}\right) & 12,5 \\ 2 & 100 & 0,08 & 14,5 \\ 2 & 200 & 0,08 & 15,0 \\ 2 & 500 & 0,10 & 18,5 \\ 3 & 100 \text { a } 110 & 0,20 & 13,5 \\ 3 & 200 \text { a } 220 & 0,08 & 20,5\end{array}$


Cuadro 2. Tolerancia permitida para matraces o balones aforados.

\section{Capacidad nominal}

$\begin{array}{ccc} & \text { Clase A } & \text { Clase B } \\ 5 & 0,02 & 0,04 \\ 10 & 0,02 & 0,04 \\ 25 & 0,03 & 0,06 \\ 50 & 0,05 & 0,10 \\ 100 & 0,08 & 0,16 \\ 200 & 0,10 & 0,20 \\ 250 & 0,12 & 0,24 \\ 500 & 0,20 & 0,40 \\ 1000 & 0,30 & 0,60 \\ 2000 & 0,50 & 1,00\end{array}$

Tolerancia $\left( \pm \mathrm{cm}^{3}\right)$

Fuente: (ASTM, 2009a).

recomienda que el equipo volumétrico de vidrio no se someta a calentamiento por encima de $150{ }^{\circ} \mathrm{C}$, pues puede provocar cambios permanentes en la capacidad de las mismas (ASTM, 2009b).

Un instrumento volumétrico cuyo coeficiente de expansión térmico sea de $25 \cdot 10^{-6}{ }^{\circ} \mathrm{C}^{-1}$ y cuya capacidad está referida a $20^{\circ} \mathrm{C}$ pero es usado a $27^{\circ} \mathrm{C}$, tendría un error adicional a la temperatura de uso del $0,02 \%$, el cual es pequeño en comparación con el límite de error volumétrico para la mayoría de los tipos de medidas. Sin embargo, el error que se introduce por la diferencia entre la temperatura de medición y la temperatura de referencia del instrumento de vidrio, depende del valor de esta diferencia y su incidencia en el resultado de la medición, de la exactitud que se pretenda alcanzar en la misma (ASTM, 2009b).

La corrección que se debe aplicar a la medición del volumen de un líquido a una temperatura $t$, con un instrumento volumétrico de vidrio calibrado a una temperatura $t_{\text {ref }}$ (por lo general $20^{\circ} \mathrm{C}$ ), se calcula a partir de ecuación (1) tomada de la información de ASTM E542-01 (ASTM, 2009b).

$$
V_{t}=V_{\text {ref }}\left[1+\alpha\left(t-t_{\text {ref }}\right)\right]
$$

Donde:

$V_{t} \quad$ volumen del instrumento a la temperatura $t$ en $\mathrm{cm}^{3}$

$V_{\text {ref }}$ volumen nominal del instrumento a la temperatura $t_{\text {ref }}$, en $\mathrm{cm}^{3}$

$\alpha \quad$ coeficiente cúbico de dilatación térmico del vidrio del que está fabricado, en ${ }^{\circ} \mathrm{C}^{-1}$

$t$ temperatura del líquido de trabajo, en ${ }^{\circ} \mathrm{C}$

$t_{\text {ref }}$ temperatura de calibración, en ${ }^{\circ} \mathrm{C}$

El error relativo en la medición del volumen debido al efecto de la temperatura sobre el vidrio del instrumento, está dado por la ecuación (2) tomada de la información de ASTM E542-01 (ASTM, 2009b).

$$
\frac{\Delta V}{V_{r e f}}=\frac{\left|V_{r e f}-V_{t}\right|}{V_{r e f}}=\alpha\left(t-t_{r e f}\right) \cdot 100
$$

Donde:

$\Delta V \quad$ diferencia entre el volumen nominal y el volumen calibrado, en $\mathrm{cm}^{3}$

$V_{\text {ref }}$ volumen nominal del instrumento a la temperatura $t_{\text {ref }}$, en $\mathrm{cm}^{3}$ 
$\alpha \quad$ coeficiente cúbico de dilatación térmico del vidrio del que está fabricado, en ${ }^{\circ} \mathrm{C}^{-1}$

$t$ temperatura del líquido de trabajo, en ${ }^{\circ} \mathrm{C}$

$t_{\text {ref }} \quad$ temperatura de calibración, en ${ }^{\circ} \mathrm{C}$

Temperatura del líquido: el volumen de los líquidos varía con la temperatura, por lo general, se trabaja con el volumen medido a la temperatura de trabajo, intervalo de $\pm 3{ }^{\circ} \mathrm{C}$. La temperatura del agua se debe medir con una exactitud de $\pm 0,1{ }^{\circ} \mathrm{C}$. No obstante, cuando se usan instrumentos volumétricos de vidrio, se debe tomar en consideración que las temperaturas de los diferentes líquidos interrelacionados para un análisis dado, estén próximas a un cierto valor común a todos cuando sus volúmenes se miden (Las autoras, 2009).

El error debido a la diferencia de temperatura del líquido a la temperatura de medición $t$ y el volumen del líquido a una temperatura de referencia $t_{r e f}$ está dado por la ecuación (3) (Elert, 1998).

$$
V_{t}=V_{r e f} \cdot \beta\left(t-t_{r e f}\right)
$$

donde:

$\beta \quad$ coeficiente cúbico de dilatación térmica del líquido con la temperatura. Para el agua, este coeficiente tiene un valor de $2,1 \cdot 10^{-4}$ ${ }^{\circ} \mathrm{C}^{-1}$.

Limpieza de la superficie del vidrio: el volumen contenido o vertido por un instrumento volumétrico de vidrio, depende de la limpieza de la superficie interna de este. Una limpieza deficiente puede dar lugar a errores debido a una forma incorrecta del menisco, lo que puede provocar dos efectos: mojado deficiente de la superficie del vidrio, o sea, la superficie del líquido toca el vidrio formando un ángulo apreciable en lugar de formar una curva tal que toque tangencialmente la pared de la medida, o un aumento del radio de curvatura del menisco, debido a contaminación de la superficie del líquido que provoca una disminución de la tensión superficial (Las autoras, 2009).
En el equipo volumétrico para verter, la falta de limpieza puede causar errores sistemáticos debido a que la capa de líquido adherida a la pared de la medida puede ser incompleta o estar irregularmente distribuida (ASTM, 2009b).

En los análisis químicos, la contaminación de los líquidos medidos debido a la limpieza deficiente del equipo volumétrico de vidrio utilizado, puede introducir un error en la composición de la disolución preparada, aun cuando la exactitud de la medición del volumen no esté significativamente afectada; por ejemplo, residuos de ácido pueden afectar la concentración de una disolución alcalina cuyo volumen se mide (Las autoras, 2009).

Para lograr una limpieza satisfactoria del equipo volumétrico de vidrio o cristalería de laboratorio se debe proceder como sigue: una vez eliminadas mecánicamente las partículas sólidas adheridas al vidrio y la grasa o el aceite mediante un disolvente adecuado, se lava la cristalería con agua y detergente agitando vigorosamente y se enjuaga varias veces con agua corriente hasta eliminar todos los vestigios de detergente; posteriormente se enjuaga con agua destilada dos o tres veces. Luego se coloca en posición vertical hacia abajo para escurrir completamente, la película de agua no debe interrumpirse si está completamente limpia (ASTM, 2009b).

Si después de esto se detecta que aún la cristalería no está completamente limpia, se debe lavar con una de las mezclas siguientes:

- una mezcla de partes iguales de $30 \mathrm{~g} / \mathrm{dm}^{3}$ de disolución de permanganato de potasio y una disolución de $1 \mathrm{~mol} / \mathrm{dm}^{3}$ de hidróxido de sodio, o

- una mezcla de hidróxido de potasio en alcohol etílico al $5 \%$.

Esta mezcla se deja reposar en la cristalería durante varias horas. Finalmente, se enjuaga abundantemente con agua del tubo y luego con agua destilada varias veces. Si la limpieza aún es deficiente, lo que se determina por la interrupción de la película de agua, se repite el procedimiento descrito. El equipo volumétrico que se limpia de esta forma y no va a ser usado de forma inmediata, debe mantenerse lleno con 
agua destilada, o bien se guarda bien cerrado para evitar su contaminación (Las autoras, 2009). Se puede obtener más información al respecto en ASTM E 542-01 (ASTM, 2009b).

Colocación del menisco: la mayor parte de los instrumentos volumétricos de vidrio emplean el principio de lectura mediante la colocación del menisco en el trazo dado de la escala. Esta puede convertirse en una importante fuente de error experimental en la determinación del volumen, por lo tanto, deben tomarse cuidados especiales para realizar una lectura correcta de la posición del menisco.

La iluminación debe ser adecuada de forma que el menisco aparezca oscuro y definido como tal. Con este propósito, este debe ser visto contra un fondo blanco y libre de iluminación indeseable. Esto puede lograrse, por ejemplo, colocando una tira de papel negro alrededor del tubo donde se forma el menisco, a una distancia no mayor de $1 \mathrm{~mm}$ por debajo del nivel donde se realiza la medición.

El error de paralaje se evita cuando la línea de graduación es de suficiente longitud para ser vista al frente y al fondo de la vasija simultáneamente. En las medidas cuyas líneas de graduación abarcan solamente la parte frontal, el paralaje puede hacerse despreciable cuando se utiliza la tira de papel negro antes descrita, teniendo cuidado de que el borde de esta se encuentre en un plano horizontal. En este caso, el ojo estará localizado de forma tal que el frente y la porción posterior del borde superior de la tira de papel se vean coincidentes.

El error que provoca el ajuste del menisco en la medición del volumen, depende de la experiencia y cuidado del observador y del diámetro del tubo donde se lee la posición del mismo. En el Cuadro 3 se dan algunos valores del error que provoca en la medición del volumen, el error lineal cometido en la posición del menisco en dependencia del diámetro interno del tubo de medición (cuerpo cilíndrico o cuello). Los valores fueron tomados de las normas ISO 384 e ISO 4787 (ISO, 1978, 1984).

Tiempo de vertido: en el equipo volumétrico de vidrio para verter, la observación del tiempo de vertido establecido, es fundamental para lograr que el volumen vertido corresponda con el valor nominal hasta el trazo dado de la escala.

Un error por la capa de líquido que se queda adherida a las paredes del equipo volumétrico de vidrio puede ocurrir también debido a una variación del tiempo normal de vertido previsto, debido a cualquier alteración (obstrucción parcial o rotura) de la punta de las pipetas o buretas. Este error no puede calcularse y afectará la exactitud de la medición realizada del volumen de forma

Cuadro 3. Errores en la posición del menisco de acuerdo al diámetro del cuello del instrumento volumétrico.

\begin{tabular}{|c|c|c|c|}
\hline \multirow[b]{2}{*}{$\begin{array}{l}\text { Error en la posición del } \\
\text { menisco }(\mathrm{mm})\end{array}$} & \multicolumn{3}{|c|}{ Valores típicos del diámetro del cuello } \\
\hline & $10 \mathrm{~mm}$ & $20 \mathrm{~mm}$ & $30 \mathrm{~mm}$ \\
\hline 0,1 & $0,01 \mathrm{~cm}^{3}$ & $0,03 \mathrm{~cm}^{3}$ & $0,07 \mathrm{~cm}$ \\
\hline 0,5 & $0,04 \mathrm{~cm}^{3}$ & $0,16 \mathrm{~cm}^{3}$ & $0,35 \mathrm{~cm}$ \\
\hline 1 & $0,08 \mathrm{~cm}^{3}$ & $0,31 \mathrm{~cm}^{3}$ & $0,71 \mathrm{~cm}$ \\
\hline 2 & $0,16 \mathrm{~cm}^{3}$ & $0,83 \mathrm{~cm}^{3}$ & $1,41 \mathrm{~cm}$ \\
\hline
\end{tabular}


significativa. Por tal motivo, cuando se utiliza una pipeta o bureta debe revisarse que la punta de ellas se encuentre en perfecto estado; así también, las bocas de los matraces y cilindros y siempre debe respetarse el tiempo de vertido señalado por el fabricante.

Método de medición: el método de calibración de la cristalería es el método gravimétrico. La capacidad de esta se determina mediante la diferencia entre la masa de la medida (si es de llenado) o de la vasija auxiliar (si es de vertido) vacía, y la masa de la misma llena con el agua destilada hasta el trazo que se comprueba. Un método estandarizado es el descrito en el apartado 11 de la norma E 542-01 (ASTM, 2009b).

Estabilización: entre las variables que afectan la densidad del agua se encuentran el aire disuelto, la pureza, los isótopos, la presión, la temperatura y la presión debido a la columna del líquido. Para propósitos de calibración se debe utilizar agua destilada, bidestilada o desionizada. Las altas concentraciones de sales, en especial sales de cobre y la materia orgánica, pueden hacer variar la densidad. El agua debe tener un $\mathrm{pH}$ neutro y su conductividad no debe exceder $1 \cdot 10^{-4} \mu \mathrm{S}$.

Cuando se realiza una calibración, lo más recomendable es utilizar una habitación con aire acondicionado para igualar las temperaturas del aire, del vidrio y del agua, con una estabilidad de $\pm 1^{\circ} \mathrm{C} / \mathrm{h}$, por lo que se recomienda mantener el agua y todos los equipos por lo menos $6 \mathrm{~h}$ antes en la misma habitación.

\section{EQUIPO, REACTIVOS Y OTROS}

El equipo utilizado y los reactivos poseían las siguientes características:

- Matraz aforado de vidrio, con capacidad de $100 \mathrm{~cm}^{3}$, clase A.

- Balanza analítica: marca Ainsworth, modelo M-220D con una resolución de $0,1 \mathrm{mg}$.

- Termómetro: marca Thermo Schneider, de 0 ${ }^{\circ} \mathrm{C}$ a $20^{\circ} \mathrm{C}$, con resolución de $0,1{ }^{\circ} \mathrm{C}$.

- Medidor eléctrico de humedad relativa y temperatura, marca TESTO.
- Agua desionizada con un pH de 6,47 a $21,6{ }^{\circ} \mathrm{C}$ y con una conductividad eléctrica de $1,4 \mu \mathrm{S}$.

- Disolución limpiadora: disolución de hidróxido de potasio $(\mathrm{KOH})$ en alcohol etílico al $5 \%$.

\section{METODOLOGÍA EXPERIMENTAL}

La metodología utilizada para la limpieza y el secado del matraz y el procedimiento de calibración utilizado fueron propuestos por los organizadores de la ronda de intercomparación (Sánchez, 2001).

\subsection{Limpieza, secado y estabilización del matraz}

Después de enjuagar el matraz por dentro y por fuera con agua destilada, se colocó una pequeña cantidad de disolución limpiadora dentro del matraz y con la tapa en su lugar, se agitó por aproximadamente dos minutos y se descartó la disolución.

Se lavó el matraz con suficiente agua del tubo, agitándolo vigorosamente cada vez hasta eliminar toda la disolución limpiadora y luego se enjuagó con agua destilada o desionizada al menos tres veces. Se secó el matraz por fuera con papel adsorbente y se colocó invertido dentro de un anillo colocado sobre un soporte, para que escurriera el agua interna, al menos diez minutos. El procedimiento debió repetirse si el matraz no quedaba perfectamente limpio a la vista.

Luego, se colocó el matraz en una estufa a $(105 \pm 5){ }^{\circ} \mathrm{C}$ por al menos $4 \mathrm{~h}$; después de ese período se desconectó el equipo y se permitió que ambos se enfriaran lentamente, hasta que alcanzó la temperatura ambiente, en ese momento se sacó el matraz de la estufa.

Tanto el agua destilada como el matraz se colocaron seis horas antes, en un cuarto con las condiciones controladas de humedad relativa y de temperatura, los valores fueron de $(23 \pm 2){ }^{\circ} \mathrm{C}$, respectivamente. En ese mismo lugar, luego de la estabilización de ambos, se realizaron las mediciones. 


\subsection{Calibración}

Se realizaron tres mediciones del volumen del matraz de vidrio de $100 \mathrm{~cm}^{3}$ utilizando el método gravimétrico descrito por Sánchez (2001) y semejante al método E 542-01 presentado en ASTM (2009b). Se registraron los datos de temperatura y humedad relativa ambiente, así como los de la temperatura del agua y las masas antes y después del llenado.

\section{ANÁLISIS Y DISCUSIÓN RESULTADOS}

\subsection{Modelo matemático}

El modelo matemático para la medición del volumen de un matraz de $100 \mathrm{~cm}^{3}$ por el método gravimétrico (4) se define en la norma ASTM E 542-01 (ASTM, 2009b), expresado aquí mediante la ecuación (4).

Donde:

$V_{20}{ }^{\circ} \mathrm{C} \quad$ volumen contenido por el instrumento, en $\mathrm{cm}^{3}$

$M_{b+a g u a} \quad$ Masa del patrón que indica la balanza (matraz lleno), en $g$

$M_{b} \quad$ Masa del patrón que indica la balanza (matraz vacío), en $g$

$\rho_{\text {agua }} \quad$ densidad del agua, en $\mathrm{g} / \mathrm{cm}^{3}$

$\rho_{\text {aire }} \quad$ densidad del aire, en $\mathrm{g} / \mathrm{cm}^{3}$

$\rho_{\text {pesas }}$ densidad de las pesas de la balanza, en $\mathrm{g} / \mathrm{cm}^{3}$

$\alpha \quad$ coeficiente cúbico de dilatación térmica, en ${ }^{\circ} \mathrm{C}^{-1}$

$t_{\text {agua }} \quad$ temperatura del agua, en ${ }^{\circ} \mathrm{C}$

$t_{\text {ref }} \quad$ temperatura de referencia, en ${ }^{\circ} \mathrm{C}$

La masa del patrón $M_{b}$ se obtiene a partir de tres mediciones de la masa de recipiente vacío, utilizando una balanza analítica en condiciones de repetibilidad, es decir, utilizando el mismo equipo de medición, en condiciones ambientales similares, con patrón similar y hecho por el mismo individuo. Lo mismo se aplica para la masa del patrón $M_{b+a g u a}$, la cual se obtiene a partir de tres mediciones de la masa de recipiente lleno de agua hasta su marca de aforo, utilizando una balanza analítica, en condiciones de repetibilidad similares a las anteriormente mencionadas.

La densidad del aire $\rho_{\text {aire }}$ es aproximada mediante la información contenida en la OIML R111 (OIML, 2004) y expresada mediante la ecuación (5).

$$
\rho_{\text {aire }}=\frac{0,34848 \cdot p-0,009\left(\frac{h r}{100}\right) \cdot e^{0,061 \cdot t_{\text {aije }}}}{1000\left(273,15+t_{\text {aire }}\right)}
$$

Donde:

$\begin{array}{ll}\rho_{\text {aire }} & \text { densidad del aire, en } \mathrm{g} / \mathrm{cm}^{3} \\ h r & \text { humedad relativa, en } \% \\ p & \text { presión atmosférica, en } \mathrm{hPa} \\ t_{\text {aire }} & \text { temperatura del aire, en }{ }^{\circ} \mathrm{C}\end{array}$

La humedad relativa $h r$, para un psicrómetro, se estima mediante la ecuación de Ferrel descrita en la norma ASTM E337-84 (ASTM, 1996 citado en Dávila \& Martines, 2006) y representada aquí por la ecuación (6).

$$
h r=100 \cdot \frac{e\left(t_{b h}\right)-A \cdot p \cdot\left(t_{b h}-t_{\text {aire }}\right)}{e\left(t_{\text {aire }}\right)}
$$

Donde:

$\begin{array}{ll}h r & \text { humedad relativa, en } \% \\ t_{b h} & \text { temperatura de bulbo húmedo, en }{ }^{\circ} \mathrm{C} \\ p & \text { presión atmosférica, en } \mathrm{hPa} \\ t_{\text {aire }} & \text { temperatura del aire, en }{ }^{\circ} \mathrm{C} \\ A & \text { coeficiente psicométrico, en }{ }^{\circ} \mathrm{C}^{-1}\end{array}$

$$
V_{20^{\circ} \mathrm{C}}=\left(M_{b+\text { agua }}-M_{b}\right)\left(\frac{1}{\rho_{\text {agua }}-\rho_{\text {aire }}}\right)\left(1-\frac{\rho_{\text {aire }}}{\rho_{\text {pesas }}}\right)\left[1-\alpha\left(t_{\text {agua }}-t_{\text {ref }}\right)\right]
$$


Cuadro 4. Términos y valores utilizados para el cálculo de la densidad del aire.

Nomenclatura

\begin{tabular}{|c|c|}
\hline$p$ & Presión atmosférica en San José, Costa Rica (hPa) \\
\hline$t_{\text {aire }}$ & Temperatura del aire o de bulbo seco $\left({ }^{\circ} \mathrm{C}\right)$ \\
\hline$t_{b h}$ & Temperatura de bulbo húmedo $\left({ }^{\circ} \mathrm{C}\right)$ \\
\hline$A$ & Coeficiente psicométrico $\left({ }^{\circ} \mathrm{C}^{-1}\right)$ \\
\hline$g_{1}$ & Coeficiente $(\mathrm{K})$ \\
\hline$g_{2}$ & Coeficiente (adim) \\
\hline$g_{3}$ & Coeficiente $\left(\mathrm{K}^{-1}\right)$ \\
\hline$g_{4}$ & Coeficiente $\left(\mathrm{K}^{-2}\right)$ \\
\hline$g_{5}$ & Coeficiente $\left(\mathrm{K}^{-3}\right)$ \\
\hline$g_{6}$ & Coeficiente $\left(\mathrm{K}^{-4}\right)$ \\
\hline$g_{7}$ & Coeficiente (adim) \\
\hline$e\left(t_{\text {aire }}\right)$ & $\begin{array}{l}\text { Presión de vapor de agua a temperatura ambiente } \\
\qquad(\mathrm{hPa})\end{array}$ \\
\hline$e\left(t_{b h}\right)$ & $\begin{array}{l}\text { Presión de vapor de agua a la temperatura de bulbo } \\
\text { húmedo (hPa) }\end{array}$ \\
\hline$h r$ & Humedad relativa (\%) \\
\hline$\rho_{\text {aire }}$ & Densidad del aire $\left(\mathrm{g} / \mathrm{cm}^{3}\right)$ \\
\hline
\end{tabular}

Magnitudes de entrada

$\begin{array}{ccc}\mathbf{1} & \mathbf{2} & \mathbf{3} \\ 880 & 880 & 880 \\ 22,00 & 20,50 & 23,75 \\ 16,50 & 14,75 & 16,00 \\ 6,6 \cdot 10^{-4} & 6,6 \cdot 10^{-4} & 6,6 \cdot 10^{-4} \\ -6,096939 \cdot 10^{3} & -6,096939 \cdot 10^{3} & -6,096939 \cdot 10^{3} \\ 21,240964 \cdot 10^{-2} & 21,240964 \cdot 10^{-2} & 21,240964 \cdot 10^{-2} \\ -2,711193 \cdot 10^{-5} & -2,711193 \cdot 10^{-5} & -2,711193 \cdot 10^{-5} \\ 1,673952 \cdot 10^{-5} & 1,673952 \cdot 10^{-5} & 1,673952 \cdot 10^{-5} \\ 0 & 0 & 0 \\ 0 & 0 & 0 \\ 2,433502 & 2,433502 & 2,433502 \\ 26,453 & 24,127 & 29,412 \\ 18,777 & 16,784 & 18,187 \\ 58,9 & 55,7 & 46,5 \\ 0,001039 & 0,001044 & 0,001033\end{array}$

Densidad estimada del aire: $0,001039 \mathrm{~g} / \mathrm{cm}^{3}$ $e\left(t_{b h}\right) \quad \begin{aligned} & \text { presión de vapor de agua a temperatura Donde: } \\ & \text { de bulbo húmedo, en } \mathrm{hPa}\end{aligned}$

$e\left(t_{\text {aire }}\right) \quad$ presión de vapor de agua a temperatura $e(T)$ presión de vapor de agua a la temperatura ambiente, en $\mathrm{hPa}$

La presión de vapor de agua puede aproximarse mediante el uso de la ecuación (7) descrita por Sonntag (Sonntag, 1990 citado en Dávila \& Martines; Dávila 2006, 2009).

$$
\ln [e(T)]=\sum_{i=1}^{6} g_{i} T^{i-2}+g_{7} \cdot \ln (T)
$$

$T \quad$ temperatura de interés, en $\mathrm{K}$

Los términos y los valores de las ecuaciones (5), (6) y (7) se definen en el Cuadro 4.

Cabe resaltar que este procedimiento para la determinación de la densidad del aire es una aproximación. Para una estimación más exacta, se puede revisar el documento CIPM-2007 de Picard, Davis, Gläser y Fujii (2008). 
Cuadro 5. Términos y valores utilizados para el cálculo de la densidad del agua.

Nomenclatura

$t_{\text {agua }}$

A

$B$

C

$D$

E

$\rho_{\text {agua }}$
Definición

Temperatura del agua $\left({ }^{\circ} \mathrm{C}\right)$

Coeficiente $\left(\mathrm{kg} / \mathrm{m}^{3}\right)$

Coeficiente $\left({ }^{\circ} \mathrm{C}^{-1} \mathrm{~kg} / \mathrm{m}^{3}\right)$

Coeficiente $\left({ }^{\circ} \mathrm{C}^{-2} \mathrm{~kg} / \mathrm{m}^{3}\right)$

Coeficiente $\left({ }^{\circ} \mathrm{C}^{-3} \mathrm{~kg} / \mathrm{m}^{3}\right)$

Coeficiente $\left({ }^{\circ} \mathrm{C}^{-4} \mathrm{~kg} / \mathrm{m}^{3}\right)$

Densidad del agua $\left(\mathrm{g} / \mathrm{cm}^{3}\right)$

Magnitudes de entrada

1

20,3

999,85308

$6,32693 \cdot 10^{-2}$

$-8,523829 \cdot 10^{-3}$

$6,943248 \cdot 10^{-5}$

$-3,821216 \cdot 10^{-7}$

0,998141
2

20,4

999,85308

$6,32693 \cdot 10^{-2}$

$-8,523829 \cdot 10^{-3}$

$6,943248 \cdot 10^{-5}$

$-3,821216 \cdot 10^{-7}$

0,998120
3

20,4

999,85308

$6,32693 \cdot 10^{-2}$

$-8,523829 \cdot 10^{-3}$

$6,943248 \cdot 10^{-5}$

$-3,821216 \cdot 10^{-7}$

0,998120

Cuadro 6. Términos y valores para la ecuación del volumen del matraz.

\section{Nomenclatura}

$M_{b}$
$M_{b+\text { agua }}$
$\rho_{\text {agua }}$
$\rho_{\text {aire }}$
$\rho_{\text {pesas }}$
$\alpha$
$t_{\text {agua }}$
$t_{\text {ref }}$
$V_{20^{\circ} \mathrm{C}}$

\section{Definición}

Masa del patrón que indica la balanza (matraz vacío) (g)

Masa del patrón que indica la balanza (matraz lleno) (g)

Densidad del agua $\left(\mathrm{g} / \mathrm{cm}^{3}\right)$

Densidad del aire $\left(\mathrm{g} / \mathrm{cm}^{3}\right)$

Densidad de las pesas de la balanza $\left(\mathrm{g} / \mathrm{cm}^{3}\right)$

Coeficiente cúbico de dilatación térmica $\left({ }^{\circ} \mathrm{C}^{-1}\right)$

Temperatura de trabajo del agua $\left({ }^{\circ} \mathrm{C}\right)$

Temperatura de referencia $\left({ }^{\circ} \mathrm{C}\right)$

Volumen contenido o vertido por el instrumento a $20^{\circ} \mathrm{C}$

$\left(\mathrm{cm}^{3}\right)$

\section{Magnitudes de entrada}

1

2

3

$79,6594 \quad 79,6598 \quad 79,6589$

$179,3856 \quad 179,3859 \quad 179,3851$

$0,998141 \quad 0,998120 \quad 0,998120$

$0,001039 \quad 0,001044 \quad 0,001033$

8,0

8,0

8,0

$0,00001 \quad 0,00001 \quad 0,00001$

20,3

20,4

20,4

20

20

20

$100,00277 \quad 100,00515 \quad 100,00424$ 
La temperatura del agua se toma con un termómetro de inmersión parcial y la densidad del agua $\rho_{\text {agua }}$ se aproxima mediante la ecuación de Kell expresada por la ecuación (8), tomada de Jones y Harris (1992). Los términos utilizados para la estimación del cálculo se presentan en el Cuadro 5.

Es importante mencionar que este procedimiento para la determinación de la densidad del agua es una aproximación. Para una estimación más exacta se puede revisar Tanaka, Girard, Davis, Peuto y Bignell (2001).

Para la construcción del equipo volumétrico se utilizan vidrios resistentes a los cambios bruscos de temperatura. Es necesario conocer el tipo de vidrio para utilizar el coeficiente de dilatación térmica. En este caso el matraz era de boro silicato y el valor del coeficiente cúbico de dilatación térmica se consideró de $\alpha=1,0 \cdot 10^{-5}{ }^{\circ} \mathrm{C}^{-1}$. Para el valor de la densidad de las pesas $\left(\rho_{\text {pesas }}\right)$ utilizadas en la medición de la masa del agua se tomó como $8000 \mathrm{~kg} / \mathrm{m}^{3}$ del certificado de calibración de las masas 12-03 del Centro Territorial de Metrología de Villa Clara, Cuba. En el Cuadro 6 se presentan, junto con las definiciones de cada uno de los términos, los valores obtenidos para tres réplicas en la determinación del volumen de un matraz de $100 \mathrm{~cm}^{3}$ a $20{ }^{\circ} \mathrm{C}$.

\subsection{Estimación de la incertidumbre de la medición}

\subsubsection{Identificación de las fuentes de incertidumbre}

Se detectaron y analizaron un total de dieciséis fuentes de incertidumbre en la medición, derivadas a partir del modelo matemático estudiado (4), las cuales se presentan a continuación y en el diagrama de espina de pescado mostrado en la Figura 1.

1. Incertidumbre por la variación de la temperatura del aire o de bulbo seco $\left(u_{l}\right)$.

2. Incertidumbre por la variación de la temperatura de bulbo húmedo $\left(u_{2}\right)$.

3. Incertidumbre por el cálculo de la humedad

$$
\rho_{\text {agua }}=\frac{A+B \cdot t_{\text {agua }}+C\left(t_{\text {agua }}\right)^{2}+D\left(t_{\text {agua }}\right)^{3} E\left(t_{\text {agua }}\right)^{4}}{1000}
$$

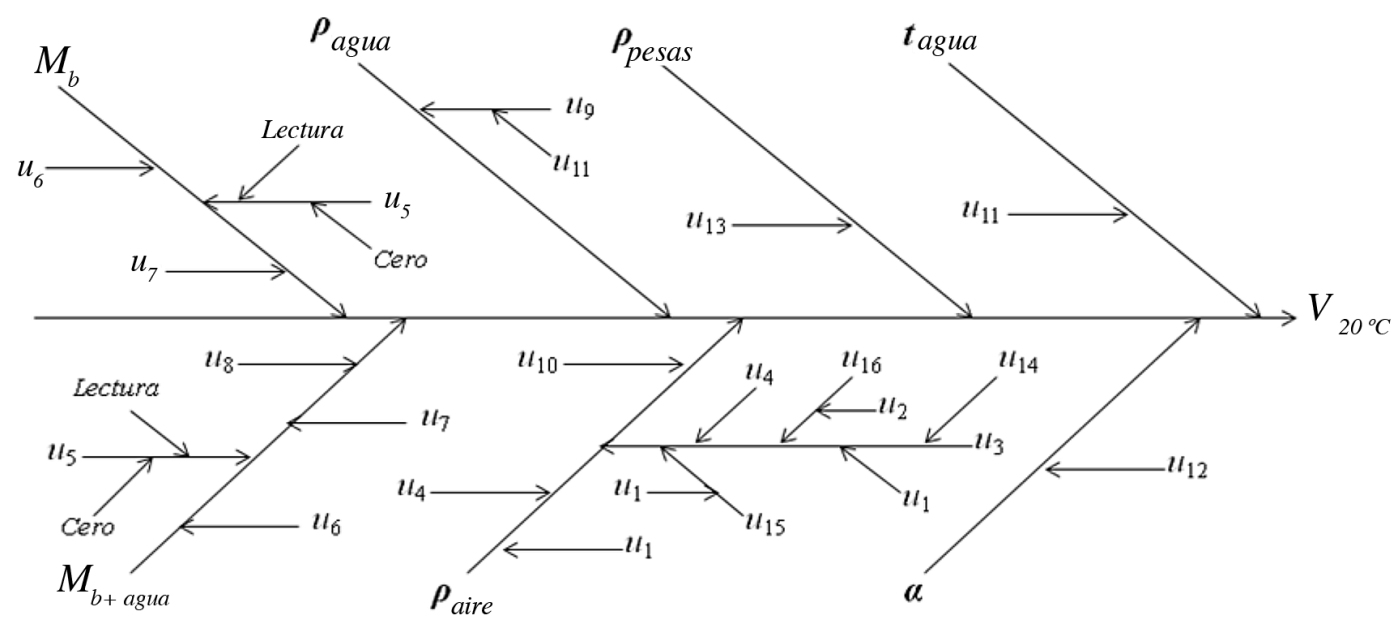

Figura 1. Diagrama de espina de pescado para las fuentes de incertidumbre identificadas. 
relativa $\left(u_{3}\right)$.

4. Incertidumbre por la estimación de la presión atmosférica $\left(u_{4}\right)$.

5. Incertidumbre por resolución de la balanza analítica $\left(u_{5}\right)$

6. Incertidumbre por repetibilidad de la balanza analítica $\left(u_{6}\right)$.

7. Incertidumbre por linealidad de la balanza analítica $\left(u_{7}\right)$.

8. Incertidumbre por observación del menisco para el matraz lleno $\left(u_{8}\right)$.

9. Incertidumbre por el cálculo de la densidad del agua $\left(u_{9}\right)$.

10. Incertidumbre por la estimación de la densidad del aire $\left(u_{10}\right)$.

11. Incertidumbre por la variación de la temperatura del agua $\left(u_{11}\right)$.

12. Incertidumbre por la estimación del coeficiente de dilatación térmica $\left(u_{12}\right)$.

13. Incertidumbre por la estimación de la densidad de la pesas de la balanza $\left(u_{13}\right)$.

14. Incertidumbre por la estimación del coeficiente psicométrico $\left(u_{14}\right)$.

15. Incertidumbre por la estimación de la presión de vapor de agua a temperatura del aire $\left(u_{15}\right)$.

16. Incertidumbre por la estimación de la presión de vapor de agua a temperatura de bulbo húmedo $\left(u_{16}\right)$.

\subsubsection{Evaluación de las fuentes de} incertidumbre estándar

Las estimaciones de incertidumbre presentadas a continuación fueran determinadas siguiendo las normativas establecidas en la GUM (1995).

\section{A) Densidad del aire}

La incertidumbre estándar asociada al error por la variación de la temperatura del aire o de bulbo seco, $u_{1}$, corresponde al cociente de $0,18{ }^{\circ} \mathrm{C}$, incertidumbre expandida reportada para el termómetro LPF-T-24 calculada en el registro LPF-R-60-4, entre su factor de cobertura $\mathrm{k}=2$, para un nivel de confianza del $95 \%$, como se muestra en la ecuación (9). $u_{1}=\frac{0,18^{\circ} \mathrm{C}}{2}=0,09^{\circ} \mathrm{C}$

La incertidumbre estándar asociada al error por la variación de la temperatura de bulbo húmedo, $u_{2}$, corresponde al cociente de $0,18{ }^{\circ} \mathrm{C}$, incertidumbre expandida reportada para el termómetro LPF-T-25 calculada en el registro LPF-R-60-5, entre su factor de cobertura $\mathrm{k}=2$, para un nivel de confianza del $95 \%$, como se muestra en la ecuación (10).

$u_{2}=\frac{0,18^{\circ} \mathrm{C}}{2}=0,09^{\circ} \mathrm{C}$

La incertidumbre por la estimación de la presión atmosférica, $u_{4}$, corresponde al cociente de $0,05 \mathrm{kPa}$, incertidumbre expandida reportada por la Oficina Nacional de Normas y Unidades de Medida (ONNUM) según el reporte LM4222000 , entre su factor de cobertura $\mathrm{k}=2$, para un nivel de confianza del $95 \%$, como se muestra en la ecuación (11).

$u_{4}=\frac{0,05 \mathrm{kPa}}{2}=0,025 \mathrm{kPa}=0,25 \mathrm{hPa}$

La incertidumbre por la estimación del coeficiente psicométrico, $u_{14}$, corresponde al cociente de $2 \cdot 10^{-5}{ }^{\circ} \mathrm{C}^{-1}$, incertidumbre expandida informada en ASTM E337-84 (ASTM, 1996 citado en Dávila \& Martines, 2006), entre su factor de cobertura $\mathrm{k}=1$, para un nivel de confianza del $68 \%$, como se muestra en la ecuación (12).

$u_{14}=\frac{2 \cdot 10^{-5}{ }^{\circ} C^{-1}}{1}=2 \cdot 10^{-5}{ }^{\circ} C^{-1}$

La incertidumbre estándar asociada al error por estimación de la presión de vapor de agua a temperatura del aire, $u_{15}$, se obtiene al aplicar la Ley de Propagación de la Incertidumbre en la ecuación (7). Tras identificar las fuentes de incertidumbre para la presión de vapor de 
agua a temperatura del aire (Figura 1), se debe proceder a estimar los coeficientes de sensibilidad necesarios. Los coeficientes de sensibilidad son valores que, al multiplicarse por las incertidumbres estándar respectivas, dan como resultado las contribuciones directas de estas sobre la incertidumbre del mensurando. Matemáticamente son definidos como la derivada parcial de cada una de las variables que conforman el modelo matemático.

En este caso, como se observa en la ecuación (7), la presión de vapor de agua a temperatura del aire $e\left(t_{\text {aire }}\right)$ solo depende de la temperatura del aire $\left(t_{\text {aire }}\right)$, por lo que solo es necesario un coeficiente de sensibilidad correspondiente a $t_{\text {aire }}$ como se muestra en la ecuación (13).

Evaluando la $t_{\text {aire }}$ promedio $\left(22,08{ }^{\circ} \mathrm{C}\right)$, $e\left(t_{\text {aire }}\right)$ promedio $(26,588 \mathrm{hPa})$ y los coeficientes respectivos (Cuadro 4), se da como resultado:

$$
\left(\frac{\partial e\left(T_{\text {aire }}\right)}{\partial T_{\text {aire }}}\right)=1,62088 \mathrm{hPa} / \mathrm{K}
$$

Aplicando la Ley de Propagación de la Incertidumbre para $e\left(t_{\text {aire }}\right), \mathrm{y}$ al poseer solo un componente, se obtiene que:

Donde:

$u^{2} c\left(e\left(t_{\text {aire }}\right)\right) \quad$ varianza estándar del error correspondiente a $e\left(T_{\text {aire }}\right), \mathrm{hPa}^{2}$
La incertidumbre estándar combinada de la presión de vapor de agua a temperatura del aire, $u_{c}\left(e\left(T_{\text {aire }}\right)\right)$, se obtiene a partir de la raíz cuadrada de la varianza estándar como se muestra en la ecuación (16).

$$
\begin{aligned}
& u_{c}\left(e\left(T_{\text {cire }}\right)\right)=u_{15}=\sqrt{\left(\frac{\partial e\left(T_{\text {aire }}\right)}{\partial T_{\text {aire }}}\right)^{2} u^{2}\left(T_{\text {aire }}\right)} \\
& u_{c}\left(e\left(T_{\text {aire }}\right)\right)=\sqrt{(1,62088 \mathrm{hPa} / \mathrm{K})^{2} \cdot(0,09 \mathrm{~K})^{2}} \\
& u_{c}\left(e\left(T_{\text {aire }}\right)\right)=u_{c}\left(e\left(T_{\text {aire }}\right)\right)=u_{15}=0,14588 \mathrm{hPa}
\end{aligned}
$$

La incertidumbre estándar asociada al error por estimación de la presión de vapor de agua a temperatura de bulbo húmedo, $u_{16}$, se obtiene al aplicar la Ley de Propagación de la Incertidumbre en la ecuación (7). Tras identificar las fuentes de incertidumbre para la presión de vapor de agua a temperatura de bulbo húmedo (Figura 1), se debe proceder a estimar los coeficientes de sensibilidad necesarios. En este caso, como se observa en la ecuación (7), presión de vapor de agua a temperatura de bulbo húmedo $e\left(T_{b h}\right)$ solo depende de la temperatura de bulbo húmedo $\left(t_{b h}\right)$, por lo que solo es necesario un coeficiente de sensibilidad correspondiente a tbh como se muestra en la ecuación (17).

Evaluando la $t_{b h}$ promedio $\left(15,75{ }^{\circ} \mathrm{C}\right)$, $\mathrm{e}\left(T_{b h}\right)$ promedio $(17,899 \mathrm{hPa})$ y los coeficientes respectivos (Cuadro 4), se da como resultado:

$$
\begin{gathered}
\left(\frac{\partial e\left(T_{\text {aire }}\right)}{\partial T_{\text {aire }}}\right)=e\left(T_{\text {aire }}\right) \cdot\left[-\frac{g_{1}}{\left(T_{\text {aire }}\right)^{2}}+g_{3}+2 g_{4}\left(T_{\text {aire }}\right)+\frac{g_{7}}{T_{\text {aire }}}\right] \\
u_{c}^{2}\left(e\left(T_{\text {aire }}\right)\right)=\left(u_{15}\right)^{2}=\left(\frac{\partial e\left(T_{\text {aire }}\right)}{\partial T_{\text {aire }}}\right)^{2}\left(u_{1}\right)^{2}=\left(\frac{\partial e\left(T_{\text {aire }}\right)}{\partial T_{\text {aire }}}\right)^{2} u^{2}\left(T_{\text {aire }}\right) \\
\left(\frac{\partial e\left(T_{b h}\right)}{\partial T_{b h}}\right)=e\left(T_{b h}\right) \cdot\left[-\frac{g_{1}}{\left(T_{b h}\right)^{2}}+g_{3}+2 g_{4}\left(T_{b h}\right)+\frac{g_{7}}{T_{b h}}\right]
\end{gathered}
$$


$\left(\frac{\partial e\left(T_{b h}\right)}{\partial T_{b h}}\right)=1,14611 \mathrm{hPa} / \mathrm{K}$

Aplicando la Ley de Propagación de la Incertidumbre para $e\left(T_{b h}\right)$, y al poseer solo un componente, se obtiene que (ver ecuación 19):

Donde:

$u_{c}^{2}\left(e\left(T_{b h}\right)\right) \quad$ varianza estándar del error correspondiente a $\mathrm{e}\left(T_{b h}\right), \mathrm{hPa}^{2}$

La incertidumbre estándar combinada de la presión de vapor de agua a de bulbo húmedo, $u_{c}\left(e\left(T_{b h}\right)\right)$, se obtiene a partir de la raíz cuadrada de la varianza estándar como se muestra en la ecuación (20).

$$
\begin{aligned}
& u_{c}\left(e\left(T_{b h}\right)\right)=u_{16}=\sqrt{\left(\frac{\partial e\left(T_{b h}\right)}{\partial T_{b h}}\right)^{2} u^{2}\left(T_{b h}\right)} \\
& u_{c}\left(e\left(T_{b h}\right)\right)=\sqrt{(1,14611 h P a / K)^{2} \cdot(0,09 K)^{2}} \\
& u_{c}\left(e\left(T_{b h}\right)\right)=u_{c}\left(e\left(T_{b h}\right)\right)=u_{16}=0,10315 \mathrm{hPa}
\end{aligned}
$$

La varianza estándar de la humedad relativa se determina calculando las incertidumbres estándar de cada sección de la ecuación (6). Primeramente, se toma la diferencia entre la temperatura del aire taire y la de bulbo húmedo $t_{b h}$. Al aplicar la Ley de Propagación de la Incertidumbre en esta función lineal, los coeficientes de sensibilidad correspondientes a dichas temperaturas se reducen a la unidad, resultando así (ver ecuación 21):

Cabe mencionar que no se presenta una correlación entre $t_{\text {aire }} \mathrm{y} t_{b h}$, ya que, físicamente, al encontrarse las fases de vapor y líquido en equilibrio, el sistema formado no posee grados de libertad, es decir, $t_{b h}$ tiene valor definido. Por su parte, la mezcla de aire con vapor de agua a presión constante sí posee un grado de libertad, permitiendo que para un valor dado de presión de vapor de agua, se pueda mantener la fase presente para una combinación infinita de temperaturas (Dávila \& Martines, 2006).

Seguidamente, se toma la sección del producto del coeficiente psicométrico A, la presión atmosférica $p$ y la diferencia de temperaturas anteriormente analizada $\left(t_{\text {aire }}-t_{b h}\right)$. Al aplicar la Ley de Propagación de la Incertidumbre en esta sección, se obtiene una incertidumbre estándar relativa para dicho producto (22). Utilizando los valores promedio de cada argumento, se obtiene:

$$
\begin{aligned}
& u_{c}^{2}\left(e\left(T_{b h}\right)\right)=\left(u_{16}\right)^{2}=\left(\frac{\partial e\left(T_{b h}\right)}{\partial T_{b h}}\right)^{2}\left(u_{2}\right)^{2}=\left(\frac{\partial e\left(T_{b h}\right)}{\partial T_{b h}}\right)^{2} u^{2}\left(T_{b h}\right) \\
& u\left(T_{\text {aire }}-T_{b h}\right)=\sqrt{u^{2}\left(T_{\text {aire }}\right)+u^{2}\left(T_{b h}\right)}=\sqrt{\left(0,09{ }^{\circ} \mathrm{C}\right)^{2}+\left(0,09{ }^{\circ} \mathrm{C}\right)^{2}} \\
& u\left(T_{\text {aire }}-T_{b h}\right)=0,127279{ }^{\circ} \mathrm{C}
\end{aligned}
$$

$$
\begin{aligned}
& \frac{u\left(A \cdot p \cdot\left(T_{\text {aire }}-T_{b h}\right)\right)}{A \cdot p \cdot\left(T_{\text {aire }}-T_{b h}\right)}=\sqrt{\left(\frac{u\left(T_{\text {aire }}-T_{b h}\right)}{\left(T_{\text {aire }}-T_{b h}\right)}\right)^{2}+\left(\frac{u(A)}{A}\right)^{2}+\left(\frac{u(p)}{p}\right)^{2}} \\
& u\left(A \cdot p \cdot\left(T_{\text {aire }}-T_{b h}\right)\right)=3,678 \mathrm{hPa} \cdot \sqrt{\left(\frac{0,127279}{6,333333}\right)^{2}+\left(\frac{2 \cdot 10^{-5}}{6,6 \cdot 10^{-4}}\right)^{2}+\left(\frac{0,25}{880,00}\right)^{2}} \\
& u\left(A \cdot p \cdot\left(T_{\text {aire }}-T_{b h}\right)\right)=0,133756 \mathrm{hPa}
\end{aligned}
$$


Posteriormente, la ecuación (23) corresponde al numerador de la ecuación (6) y se utiliza para estimar su incertidumbre estándar. Al aplicar la Ley de Propagación de la Incertidumbre en esta función lineal, los coeficientes de sensibilidad correspondientes a los argumentos se reducen a la unidad, resultando así la ecuación (24). Se utilizando los valores promedio de cada argumento.

num $=e\left(T_{b h}\right)-A \cdot p \cdot\left(t_{\text {aire }}-t_{b h}\right)=14,222 h P a$

$$
\begin{aligned}
& u(\text { num })=\sqrt{u_{c}^{2}\left(e\left(T_{b h}\right)\right)+u^{2}\left(A \cdot p \cdot\left(t_{\text {aire }}-t_{b h}\right)\right)} \\
& u(\text { num })=\sqrt{(0,10315 \mathrm{hPa})^{2}+(0,133756 \mathrm{hPa})^{2}} \\
& u(\text { num })=0,1689 \mathrm{hPa}
\end{aligned}
$$

Por último, se estima la incertidumbre estándar de la humedad relativa aplicando la Ley de Propagación de la Incertidumbre en el cociente entre el numerador (num) y el denominador $\left(e\left(T_{\text {aire }}\right)\right)$. Se obtiene una incertidumbre estándar relativa para dicho cociente (25), la cual se estima utilizando los valores promedio de cada argumento.
La incertidumbre por la estimación de la densidad del aire, $u_{10}$, corresponde al cociente de $0,000003 \mathrm{~g} / \mathrm{cm}^{3}$, incertidumbre expandida reportada en el PTB-MA-311, entre su factor de cobertura $\mathrm{k}=2$, para un nivel de confianza del $95 \%$, como se ilustra en la ecuación (26).

$$
u_{10}=\frac{0,000003}{2} \mathrm{~g} / \mathrm{cm}^{3}=0,0000015 \mathrm{~g} / \mathrm{cm}^{3}
$$

Así, la varianza estándar correspondiente a $\rho_{\text {aire }}$ se obtiene al aplicar la Ley de Propagación de la Incertidumbre en la ecuación (5). Tras identificar las fuentes de incertidumbre para la densidad del aire (Figura 1), se debe proceder a calcular los coeficientes de sensibilidad pertinentes. En este caso, como se nota en (5), $\rho_{\text {aire }}$ depende de $t_{\text {aire }}, h r$ y $p$, por lo que es necesario calcular tres coeficientes. El coeficiente de sensibilidad para la incertidumbre asociada a $t_{\text {aire }}$ está dado por la ecuación (27).

El coeficiente de sensibilidad para la incertidumbre asociada a $h r$ está dado por la ecuación (28)

$$
\left(\frac{\partial \rho_{\text {aire }}}{\partial h r}\right)=\frac{-0,009 \cdot e^{0,061 \cdot t_{\text {aire }}}}{1000\left(273,15+t_{\text {aire }}\right)} \cdot \frac{1}{100}
$$


El coeficiente de sensibilidad para la incertidumbre asociada a $p$ está dado por la ecuación (29).

$$
\left(\frac{\partial \rho_{\text {aire }}}{\partial p}\right)=\frac{0,34848}{1000\left(273,15+t_{\text {aire }}\right)}
$$

$$
\begin{aligned}
& \left(\frac{\partial \rho_{\text {aire }}}{\partial t_{\text {aire }}}\right)=-3,521982 \cdot 10^{-6} \mathrm{~g} / \mathrm{cm}^{3} \cdot{ }^{\circ} \mathrm{C} \\
& \left(\frac{\partial \rho_{\text {aire }}}{\partial h r}\right)=-1,172262 \cdot 10^{-9} \mathrm{~g} / \mathrm{cm}^{3} \\
& \left(\frac{\partial \rho_{\text {aire }}}{\partial p}\right)=1,180368 \cdot 10^{-6} \mathrm{~g} /\left(\mathrm{cm}^{3} \cdot \mathrm{hPa}\right)
\end{aligned}
$$

Evaluando los valores promedio de cada variable considerada en el Cuadro 4, en cada coeficiente de sensibilidad, se obtiene que:
Aplicando la Ley de Propagación de la Incertidumbre para $\rho_{\text {aire }}$, se obtiene la ecuación (33).

$$
u_{c}^{2}\left(\rho_{\text {aire }}\right)=\left(\frac{\partial \rho_{\text {aire }}}{\partial t_{\text {aire }}}\right)^{2}\left(u_{1}\right)^{2}+\left(\frac{\partial \rho_{\text {aire }}}{\partial h r}\right)^{2}\left(u_{3}\right)^{2}+\left(\frac{\partial \rho_{\text {aire }}}{\partial p}\right)^{2}\left(u_{4}\right)^{2}+\left(u_{10}\right)^{2}
$$

Cuadro 7. Términos y valores para la incertidumbre estándar combinada del volumen del matraz.

\section{Nomenclatura}

$M_{b}$
$M_{b+\text { agua }}$
$\rho_{\text {agua }}$
$\rho_{\text {aire }}$
$t_{\text {agua }}$
$\alpha$
$\rho_{\text {pesas }}$

\section{Definición}

Masa del patrón que indica la balanza (matraz vacío)

Masa del patrón que indica la balanza (matraz lleno)

Densidad del agua

Densidad del aire

Temperatura de trabajo del agua

Coeficiente cúbico de dilatación térmica
Densidad de las pesas
de la balanza

Densidad de las pesas
de la balanza

\section{Valor promedio}

$79,6594 \mathrm{~g}$

$179,3869 \mathrm{~g}$

$0,998127 \mathrm{~g} / \mathrm{cm}^{3}$

$0,001039 \mathrm{~g} / \mathrm{cm}^{3}$

$20,37^{\circ} \mathrm{C}$

$0,00001{ }^{\circ} \mathrm{C}^{-1}$

$8,0 \mathrm{~g} / \mathrm{cm}^{3}$

\section{Incertidumbre} estándar

$0,000072 \mathrm{~g}$

$1,002786 \mathrm{~cm}^{3} / \mathrm{g}$

$0,01530 \mathrm{~g}$

$-1,002786 \mathrm{~cm}^{3} / \mathrm{g}$

$5,21 \cdot 10^{-9} \mathrm{~cm}^{6}$

$1,0524 \cdot 10-5 \mathrm{~g} / \mathrm{cm}^{3}$

$1,56 \cdot 10-6 \mathrm{~g} / \mathrm{cm}^{3}$

$0,05{ }^{\circ} \mathrm{C}$

$-0,001 \mathrm{~cm}^{3} /{ }^{\circ} \mathrm{C}$

$5,77 \cdot 10-6{ }^{\circ} \mathrm{C}^{-1}$

$-37,002 \mathrm{~cm}^{3} \cdot{ }^{\circ} \mathrm{C}$

$4,56 \cdot 10^{-8} \mathrm{~cm}^{6}$
$0,019999 \mathrm{~g} / \mathrm{cm}^{3}$
$0,001623 \mathrm{~cm}^{6} / \mathrm{g}$

Contribución

$2,34 \cdot 10^{-4} \mathrm{~cm}^{6}$

$1,11 \cdot 10^{-6} \mathrm{~cm}^{6}$

$1,88 \cdot 10^{-8} \mathrm{~cm}^{6}$ $2,5 \cdot 10^{-9} \mathrm{~cm}^{6}$ Incertidumbre estándar combinada para el volumen estimado del matraz a $20{ }^{\circ} \mathrm{C}=0,015381 \mathrm{~cm}^{3}$ 
donde:

$u_{c}^{2}\left(\rho_{\text {aire }}\right) \quad$ varianza estándar de a $\rho_{\text {aire }},\left(\mathrm{g} / \mathrm{cm}^{3}\right)^{2}$

La incertidumbre estándar combinada de la densidad del aire, $u_{c}\left(\rho_{\text {aire }}\right)$, se obtiene a partir de la raíz cuadrada de la varianza estándar, aplicando la ecuación (34).

En este punto hay que mencionar que se desprecian las contribuciones por correlación entre la presión atmosférica, la temperatura ambiente y la humedad relativa.

B) Masa del patrón que indica la balanza (matraz vacío)

La incertidumbre estándar asociada al error por resolución de la balanza analítica, $u_{5}$, considerando que obedece a una distribución rectangular, corresponde a la mitad del valor reportado por el fabricante entre la raíz cuadrada de 3 , como se presenta en la ecuación (35):

$u_{5}=\frac{0,0001 g}{2 \sqrt{3}}=0,0000288 g$
Se debe tomar en consideración que el error por resolución se presenta tanto al tomar la medición como al tomar el cero como valor de partida, ya que la lectura tomada es en realidad una diferencia entre la masa inicial (cero) y la masa final. Esto causa que esta incertidumbre deba ser considerada dos veces a la hora de realizar los cálculos respectivos.

$\mathrm{La}$ incertidumbre estándar asociada al error por repetibilidad de la balanza analítica, $u_{6}$, considerando que obedece a una distribución rectangular, corresponde al valor reportado por el fabricante entre la raíz cuadrada de tres, tal y como se muestra en la ecuación (36).

$u_{6}=\frac{0,0001 g}{\sqrt{3}}=0,0000577 \mathrm{~g}$

La incertidumbre estándar asociada al error por linealidad de la balanza analítica, $u_{7}$, considerando que obedece a una distribución rectangular, corresponde al valor reportado por el fabricante entre la raíz cuadrada de tres, tal y como se observa en la ecuación (37).

$u_{7}=\frac{0,00002 g}{\sqrt{3}}=0,0000115 \mathrm{~g}$

$$
\begin{aligned}
& u_{c}\left(\rho_{\text {aire }}\right)=\sqrt{\left(\frac{\partial \rho_{\text {aire }}}{\partial t_{\text {aire }}}\right)^{2}\left(u_{1}\right)^{2}+\left(\frac{\partial \rho_{\text {aire }}}{\partial h r}\right)^{2}\left(u_{3}\right)^{2}+\left(\frac{\partial \rho_{\text {aire }}}{\partial p}\right)^{2}\left(u_{4}\right)^{2}+\left(u_{10}\right)^{2}} \\
& \left(\frac{\partial \rho_{\text {aire }}}{\partial t_{\text {aire }}}\right)^{2}\left(u_{1}\right)^{2}=\left[\left(-3,521982 \cdot 10^{-6}\right)^{2} \cdot(0,09)^{2}\right]\left(\mathrm{g} / \mathrm{cm}^{3}\right)^{2}=1,00475 \cdot 10^{-13}\left(\mathrm{~g} / \mathrm{cm}^{3}\right)^{2} \\
& \left(\frac{\partial \rho_{\text {aire }}}{\partial h r}\right)^{2}\left(u_{3}\right)^{2}=\left[\left(-1,172262 \cdot 10^{-9}\right)^{2} \cdot(0,669)^{2}\right]\left(\mathrm{g} / \mathrm{cm}^{3}\right)^{2}=6,02943 \cdot 10^{-19}\left(\mathrm{~g} / \mathrm{cm}^{3}\right)^{2} \\
& \left(\frac{\partial \rho_{\text {aire }}}{\partial p}\right)^{2}\left(u_{4}\right)^{2}=\left[\left(1,180368 \cdot 10^{-6}\right)^{2} \cdot(0,25)^{2}\right]\left(\mathrm{g} / \mathrm{cm}^{3}\right)^{2}=8,707929 \cdot 10^{-14}\left(\mathrm{~g} / \mathrm{cm}^{3}\right)^{2} \\
& \left(u_{10}\right)^{2}=\left(1,5 \cdot 10^{-6}\right)^{2}\left(\mathrm{~g} / \mathrm{cm}^{3}\right)^{2}=2,25 \cdot 10^{-12}\left(\mathrm{~g} / \mathrm{cm}^{3}\right)^{2} \\
& u_{c}\left(\rho_{\text {aire }}\right)=\sqrt{1,00475 \cdot 10^{-13}+6,02943 \cdot 10^{-19}+8,707929 \cdot 10^{-14}+2,25 \cdot 10^{-12}} \mathrm{~g} / \mathrm{cm}^{3} \\
& u_{c}\left(\rho_{\text {aire }}\right)=1,56 \cdot 10^{-6} \mathrm{~g} / \mathrm{cm}^{3}
\end{aligned}
$$


Así, la varianza estándar correspondiente a $M_{b}$ se determina a partir de los cuadrados de cada una de las contribuciones a la incertidumbre de esta, como se ilustra en la ecuación (38).

$u_{c}^{2}\left(M_{b}\right)=2\left(u_{5}\right)^{2}+\left(u_{6}\right)^{2}+\left(u_{7}\right)^{2}$

Donde:

$u_{c}^{2}\left(M_{b}\right) \quad$ varianza estándar de $M_{b}, \mathrm{~g}^{2}$

La incertidumbre estándar combinada de la masa del patrón que indica la balanza (matraz vacío), $u_{c}\left(M_{b}\right)$, se obtiene a partir de la raíz cuadrada de la varianza estándar presentada en la ecuación (39).

C) Masa del patrón que indica la balanza (matraz lleno)

La incertidumbre estándar asociada al error por observación del menisco para el matraz lleno, $u_{8}$, considerando que obedece a una distribución rectangular, corresponde al diferencial de masa de agua perteneciente al menisco entre la raíz cuadrada de tres, como se presenta en la ecuación (40).

$$
u_{g}=\frac{\Delta m}{\sqrt{3}}
$$

Para calcular el diferencial de masa, se procede a aproximarlo mediante el uso de las ecuaciones
(41) y (42), para el cálculo del volumen de un cilindro y la densidad del agua, respectivamente.

$$
\Delta V=\pi\left(\frac{d^{2}}{4}\right) \Delta h \cdot 10^{-3}
$$

$$
\rho_{\text {agua }}=\left(\frac{\Delta m}{\Delta V}\right)
$$

Donde:

$\Delta m \quad$ diferencial de masa del menisco, $\mathrm{g}$

$\Delta V$ diferencial de volumen del menisco, $\mathrm{cm}^{3}$

d diámetro interno del cuello de un matraz de $100 \mathrm{~cm}^{3}, 13 \mathrm{~mm}$ (ISO 1042, tabla 10)

$\Delta h \quad$ diferencial de altura del menisco, $0,2 \mathrm{~mm}$ (ISO 384)

$\rho_{\text {agua }} \quad$ densidad del agua, $0,998127 \mathrm{~g} / \mathrm{cm}^{3}$ (Cuadro 5)

$\mathrm{Al}$ despejar el diferencial de volumen de la ecuación (42) y sustituirlo en la ecuación (41), se obtiene una expresión para el diferencial de masa de la forma que se presenta en la ecuación (43).

$$
\Delta m=\pi\left(\frac{d^{2}}{4}\right) \cdot \Delta h \cdot \rho_{\text {agua }} \cdot 10^{-3}
$$

Al unir la ecuación (40) con la (43) y sustituir por los valores numéricos correspondientes se obtiene la ecuación (44).

$$
\begin{aligned}
& u_{c}\left(M_{b}\right)=\sqrt{u_{c}^{2}\left(M_{b}\right)}=\sqrt{2\left(u_{5}\right)^{2}+\left(u_{6}\right)^{2}+\left(u_{7}\right)^{2}} \\
& u_{c}\left(M_{b}\right)=\sqrt{2(0,0000288 g)^{2}+(0,0000577 g)^{2}+(0,0000115 g)^{2}} \\
& u_{c}\left(M_{b}\right)=0,000072 g
\end{aligned}
$$

$$
u_{g}=\frac{\pi\left(\frac{13^{2}}{4} \mathrm{~mm}^{2}\right) \cdot 0,2 \mathrm{~mm} \cdot 0,998127 \mathrm{~g} / \mathrm{cm}^{3} \cdot 10^{-3}}{\sqrt{3}}=\frac{0,02650 \mathrm{~g}}{\sqrt{3}}=0,0153 \mathrm{~g}
$$


Cabe mencionar que se podría tomar la incertidumbre por el menisco como un diferencial de volumen y agregarlo como un factor de incertidumbre separado de la masa del matraz lleno.

Las incertidumbres estándar asociadas a los errores por resolución, repetibilidad y linealidad de la balanza analítica, $u_{5}, u_{6}$ y $u_{7}$, fueron calculadas en (35), (36) y (37) respectivamente.

Así, la varianza estándar correspondiente a $M_{b+a g u a}$ se determina a partir de los cuadrados de cada una de las contribuciones a la incertidumbre de esta, como se observa en la ecuación (45).

$u_{c}^{2}\left(M_{b+a g u a}\right)=2\left(u_{5}\right)^{2}+\left(u_{6}\right)^{2}+\left(u_{7}\right)^{2}+\left(u_{8}\right)^{2}$

Donde:

$u_{c}^{2}\left(M_{b+a g u a}\right) \quad$ varianza estándar de $M_{b+a g u a}, \mathrm{~g}^{2}$

La incertidumbre estándar combinada de la patrón que indica la balanza (matraz lleno), $u_{c}\left(M_{b+a g u a}\right)$, se obtiene a partir de la raíz cuadrada de la varianza estándar, como se muestra en la ecuación (46).

\section{D) Temperatura del agua}

La incertidumbre estándar asociada al error por la variación de la temperatura del agua, $u_{l l}$, corresponde al cociente de $0,10{ }^{\circ} \mathrm{C}$, incertidumbre expandida tomada del certificado 168-2001 T, del LABCAL/MACNOR de calibración del termómetro utilizado, entre su factor de cobertura $\mathrm{k}=2$, para un nivel de confianza del $95 \%$, como se muestra en la ecuación (47).

$u_{11}=\frac{0,10^{\circ} \mathrm{C}}{2}=0,05^{\circ} \mathrm{C}$

Al poseer solamente un componente, la incertidumbre estándar combinada de la temperatura del agua, $u\left(t_{\text {agua }}\right)$, es igual a $\left(u_{11}\right)$.

$u\left(t_{\text {agua }}\right)=u_{11}=0,05^{\circ} \mathrm{C}$

\section{E) Densidad del agua}

La incertidumbre estándar asociada al error por el cálculo de la densidad del agua, $u_{9}$, se obtiene al aplicar la Ley de Propagación de la Incertidumbre en la ecuación (8). Tras identificar las fuentes de incertidumbre para la densidad del agua (Figura 1), se debe proceder a estimar los coeficientes de sensibilidad necesarios. En este caso, como se observa en la ecuación (8), la densidad del agua $\left(\rho_{\text {agua }}\right)$ solo depende de la temperatura del agua $\left(t_{\text {agua }}\right)$, por lo que solo es necesario un coeficiente de sensibilidad correspondiente a tagua como se muestra en la ecuación (49).

Evaluando la $t_{\text {agua }}$ promedio $\left(20,37^{\circ} \mathrm{C}\right)$ y los coeficientes (Cuadro 5), se da como resultado:

$$
\begin{aligned}
& u_{c}\left(M_{b+\text { agua }}\right)=\sqrt{u_{c}^{2}\left(M_{b+\text { agua }}\right)}=\sqrt{2\left(u_{5}\right)^{2}+\left(u_{6}\right)^{2}+\left(u_{7}\right)^{2}+\left(u_{8}\right)^{2}} \\
& u_{c}\left(M_{b+\text { agua }}\right)=\sqrt{2(0,0000288 \mathrm{~g})^{2}+(0,0000577 \mathrm{~g})^{2}+(0,0000115 \mathrm{~g})^{2}+(0,0153 \mathrm{~g})^{2}} \\
& u_{c}\left(M_{b+\text { agua }}\right)=0,01530 \mathrm{~g}
\end{aligned}
$$

$$
\left(\frac{\delta \rho_{\text {agua }}}{\delta t_{\text {agua }}}\right)=\frac{B+2 \cdot C \cdot t_{\text {agua }}+3 \cdot D \cdot\left(t_{\text {agua }}\right)^{2}+4 \cdot E \cdot\left(t_{\text {agua }}\right)^{2}}{1000}
$$


$\left(\frac{d \rho_{\text {agua }}}{d t_{\text {agua }}}\right)=-2,105 \cdot 10^{-4} \mathrm{~g} /\left({ }^{\circ} \mathrm{C} \cdot \mathrm{cm}^{3}\right)$

Aplicando la Ley de Propagación de la Incertidumbre para $\rho_{\text {agua }}$, y al poseer solo un componente, se obtiene que:

Donde:

$u_{c}^{2}\left(\rho_{\text {agua }}\right)$ varianza estándar del error correspondiente a $\rho_{\text {agua }},\left(\mathrm{g} / \mathrm{cm}^{3}\right)^{2}$

La incertidumbre estándar combinada de la densidad del agua, $u_{c}\left(\rho_{\text {agua }}\right)$, se obtiene a partir de la raíz cuadrada de la varianza estándar como se muestra en la ecuación (52).

\section{F) Coeficiente de dilatación térmica}

La incertidumbre estándar asociada al error por la estimación del coeficiente de dilatación térmica, $u_{12}$, considerando que obedece a una distribución rectangular, corresponde al valor de $1 \cdot 10^{-5}{ }^{\circ} \mathrm{C}^{-1}$, tomado del ENA-034-01, entre la raíz cuadrada de 3 , tal y como se ilustra en la ecuación (53).

$u_{12}=\frac{1 \cdot 10^{-5}}{\sqrt{3}}{ }^{\circ} C^{-1}=5,77 \cdot 10^{-6}{ }^{\circ} C^{-1}$
Al solamente poseer un componente, la incertidumbre estándar combinada del coeficiente cúbico de dilatación térmica, $u(\alpha)$, es igual a $u_{12}$.

$u(\alpha)=u_{12}=5,77 \cdot 10^{-6}{ }^{\circ} C^{-1}$

G) Densidad de los patrones de masa de la balanza

La incertidumbre estándar asociada al error por la estimación de la densidad de los patrones de masas de la balanza, $u_{13}$, considerando que obedece a una distribución rectangular, corresponde al valor de $0,03464 \mathrm{~g} / \mathrm{cm}^{3}$, tomado de la norma alemana, entre la raíz cuadrada de 3 , tal y como se ilustra en la ecuación (55).

$$
u_{13}=\frac{0,03464}{\sqrt{3}} \mathrm{~g} / \mathrm{cm}^{3}=0,019999 \mathrm{~g} / \mathrm{cm}^{3}
$$

Al solamente poseer un componente, la incertidumbre estándar combinada del coeficiente de dilatación térmica, $u\left(\rho_{\text {pesas }}\right)$, es igual a la incertidumbre asociada al error por la estimación de la densidad de las pesas de la balanza, $u_{13}$ :

$u\left(\rho_{\text {pesas }}\right)=u_{13}=0,019999 \mathrm{~g} / \mathrm{cm}^{3}$

$$
\begin{aligned}
& u_{c}^{2}\left(\rho_{\text {agua }}\right)=\left(u_{9}\right)^{2}=\left(\frac{d \rho_{\text {agua }}}{d t_{\text {agua }}}\right)^{2}\left(u_{11}\right)^{2}=\left(\frac{d \rho_{\text {agua }}}{d t_{\text {agua }}}\right)^{2} u^{2}\left(t_{\text {agua }}\right) \\
& u_{c}\left(\rho_{\text {agua }}\right)=u_{9}=\sqrt{\left(\frac{d \rho_{\text {agua }}}{d t_{\text {agua }}}\right)^{2} u^{2}\left(t_{\text {agua }}\right)} \\
& u_{c}\left(\rho_{\text {agua }}\right)=\sqrt{\left(-2,105 \cdot 10^{-4} \mathrm{~g} /\left({ }^{\circ} \mathrm{C} \cdot \mathrm{cm}^{3}\right)\right)^{2} \cdot\left(0,05^{\circ} \mathrm{C}\right)^{2}} \\
& u_{c}\left(\rho_{\text {agua }}\right)=1,0524 \cdot 10^{-5} \mathrm{~g} / \mathrm{cm}^{3}
\end{aligned}
$$




\subsubsection{Coeficientes de sensibilidad}

Como se mencionó en el apartado de la evaluación de las fuentes de incertidumbre estándar, los coeficientes de sensibilidad son valores que, junto a las incertidumbres estándar, dan como resultado las contribuciones directas de estas sobre la incertidumbre del mensurando. A continuación se presentan los coeficientes de sensibilidad de cada una de las variables presentes en la ecuación (4).

El coeficiente de sensibilidad para la incertidumbre asociada a $M_{b+a g u a}$ está dado por la ecuación (57).

El coeficiente de sensibilidad para la incertidumbre asociada a $M_{b}$ está dado por la ecuación (58).

El coeficiente de sensibilidad para la incertidumbre asociada a $\rho_{\text {agua }}$ está dado por la ecuación (59).
El coeficiente de sensibilidad para la incertidumbre asociada a $\rho_{\text {aire }}$ está dado por la ecuación (60).

El coeficiente de sensibilidad para la incertidumbre asociada a $t_{\text {agua }}$ está dado por la ecuación (61).

El coeficiente de sensibilidad para la incertidumbre asociada a $\alpha$ está dado por la ecuación (62).

$$
\left(\frac{\partial V_{20^{\circ} \mathrm{C}}}{\partial \alpha}\right)=\frac{\left(M_{b+a g u a}-M_{b}\right)}{\left(\rho_{\text {agua }}-\rho_{\text {aire }}\right)}\left(1-\frac{\rho_{\text {aire }}}{\rho_{\text {pesas }}}\right)\left(t_{\text {agua }}-t_{\text {ref }}\right)
$$

El coeficiente de sensibilidad para la incertidumbre asociada a $\rho_{\text {pesas }}$ está dado por la ecuación (63).

$$
\begin{gathered}
\left(\frac{\partial V_{20^{\circ} \mathrm{C}}}{\partial M_{b+\text { agua }}}\right)=\left(\frac{1}{\rho_{\text {agua }}-\rho_{\text {aire }}}\right)\left(1-\frac{\rho_{\text {aire }}}{\rho_{\text {pesas }}}\right)\left[1-\alpha\left(t_{\text {agua }}-t_{\text {ref }}\right)\right] \\
\left(\frac{\partial V_{20^{\circ} \mathrm{C}}}{\partial M_{b}}\right)=-\left(\frac{1}{\rho_{\text {agua }}-\rho_{\text {aire }}}\right)\left(1-\frac{\rho_{\text {aire }}}{\rho_{\text {pesas }}}\right)\left[1-\alpha\left(t_{\text {agua }}-t_{\text {ref }}\right)\right] \\
\left(\frac{\partial V_{20^{\circ} \mathrm{C}}}{\partial \rho_{\text {agua }}}\right)=\frac{-\left(M_{b+\text { agua }}-M_{b}\right)}{\left(\rho_{\text {agua }}-\rho_{\text {aire }}\right)^{2}} \cdot\left(1-\frac{\rho_{\text {aire }}}{\rho_{\text {pesas }}}\right) \cdot\left[1-\alpha\left(t_{\text {agua }}-t_{\text {ref }}\right)\right] \\
\left(\frac{\partial V_{20^{\circ} \mathrm{C}}}{\partial \rho_{\text {aire }}}\right)=\frac{\left(M_{b+\text { agua }}-M_{b}\right)}{\left(\rho_{\text {agua }}-\rho_{\text {aire }}\right)}\left[\frac{\left(1-\frac{\rho_{\text {aire }}}{\rho_{\text {pesas }}}\right)}{\left(\rho_{\text {agua }}-\rho_{\text {aire }}\right)}-\frac{1}{\rho_{\text {pesas }}}\right]\left[1-\alpha\left(t_{\text {agua }}-t_{\text {ref }}\right)\right] \\
\left(\frac{\partial V_{20^{\circ} \mathrm{C}}}{\partial t_{\text {agua }}}\right)=\frac{\left(M_{b+\text { agua }}-M_{b}\right)}{\left(\rho_{\text {agua }}-\rho_{\text {aire }}\right)}\left(1-\frac{\rho_{\text {aire }}}{\rho_{\text {pesas }}}\right)(-\alpha) \\
\left(\frac{\partial V_{20^{\circ} C}}{\partial \rho_{\text {pesas }}}\right)=\frac{\left(M_{b+\text { agua }}-M_{b}\right)}{\left(\rho_{\text {agua }}-\rho_{\text {aire }}\right)} \cdot\left[1-\alpha\left(t_{\text {agua }}-t_{\text {ref }}\right)\right] \cdot \frac{\rho_{\text {aire }}}{\left(\rho_{\text {pesas }}\right)^{2}}
\end{gathered}
$$


Evaluando los valores promedio de cada variable considerada (Cuadro 6) en cada coeficiente de sensibilidad, se obtiene que:

$\left(\frac{\partial V_{20^{\circ} \mathrm{C}}}{\partial M_{b+\text { agua }}}\right)=1,002786 \mathrm{~cm}^{3} / \mathrm{g}$

$\left(\frac{\partial V_{20^{\circ} \mathrm{C}}}{\partial M_{b}}\right)=-1,002786 \mathrm{~cm}^{3} / \mathrm{g}$

$\left(\frac{\partial V_{20^{\circ} \mathrm{C}}}{\partial \rho_{\text {agиа }}}\right)=-100,29610\left(\mathrm{~cm}^{3}\right)^{2} / \mathrm{g}$

$\left(\frac{\partial V_{20^{\circ} \mathrm{C}}}{\partial \rho_{\text {aire }}}\right)=87,79397\left(\mathrm{~cm}^{3}\right)^{2} / \mathrm{g}$

$\left(\frac{\partial V_{20^{\circ} \mathrm{C}}}{\partial t_{\text {agua }}}\right)=-0,001 \mathrm{~cm}^{3} /{ }^{\circ} \mathrm{C}$

$\left(\frac{\partial V_{20^{\circ} \mathrm{C}}}{\partial \alpha}\right)=-37,002 \mathrm{~cm}^{3} \cdot{ }^{\circ} \mathrm{C}$

$$
\left(\frac{\partial V_{20^{\circ} \mathrm{C}}}{\partial \rho_{\text {pesas }}}\right)=0,001623\left(\mathrm{~cm}^{3}\right)^{2} / \mathrm{g}
$$

\subsubsection{Incertidumbre combinada estándar}

La varianza estándar (71) se calcula a partir de los cuadrados de cada una de las contribuciones a la incertidumbre del mensurando. Las contribuciones son el resultado del producto de cada incertidumbre por su respectivo coeficiente de sensibilidad.

Donde:

$u_{c}^{2}\left(V_{20}{ }^{\circ} \mathrm{C}\right) \quad$ varianza estándar del error correspondiente a $V_{20^{\circ} \mathrm{C}},\left(\mathrm{cm}^{3}\right)^{2}$

La incertidumbre estándar combinada del volumen contenido por el matraz a $20{ }^{\circ} \mathrm{C}$, $u_{c}\left(V_{20}{ }^{\circ} \mathrm{C}\right)$, se obtiene a partir de la raíz cuadrada de la varianza estándar calculada mediante la ecuación (72).

A manera de resumen, se presenta el Cuadro 7, para las variables consideradas, su valor promedio, incertidumbre, coeficiente de sensibilidad y contribución a la incertidumbre global en la determinación del volumen de un matraz de $100 \mathrm{~cm}^{3}$ a $20{ }^{\circ} \mathrm{C}$. Cabe mencionar que para esta estimación de incertidumbre se despreciaron las contribuciones por correlación entre la temperatura del agua y la densidad de la misma, además de la correlación entre la masa del matraz vacío y la masa del matraz lleno.

\subsubsection{Incertidumbre expandida}

La incertidumbre expandida del volumen contenido por el matraz a $20{ }^{\circ} \mathrm{C}, U_{V_{20}{ }^{\circ} \mathrm{C}}$, se determina a partir de la ecuación (73).

$U_{V_{20^{\circ} \mathrm{C}}}= \pm u_{c}\left(V_{20^{\circ} \mathrm{C}}\right) \cdot k$

Donde:

$U_{V_{20}{ }^{\circ} \mathrm{C}} \quad$ incertidumbre expandida del volumen del matraz a $20^{\circ} \mathrm{C}$

$k$ factor de cobertura para un nivel de confianza del $95 \%$.

$$
u_{c}^{2}\left(V_{20^{\circ} \mathrm{C}}\right)=\left[\begin{array}{l}
\left(\frac{\partial V_{20^{\circ} \mathrm{C}}}{\partial M_{b+\text { ggua }}}\right)^{2} u_{c}^{2}\left(M_{b+\text { agua }}\right)+\left(\frac{\partial V_{20^{\circ} \mathrm{C}}}{\partial M_{b}}\right)^{2} u_{c}^{2}\left(M_{b}\right)+\left(\frac{\partial V_{20^{\circ} \mathrm{C}}}{\partial \rho_{\text {agua }}}\right)^{2} u_{c}^{2}\left(\rho_{\text {agua }}\right)+ \\
\left(\frac{\partial V_{20^{\circ} \mathrm{C}}}{\partial \rho_{\text {aire }}}\right)^{2} u_{c}^{2}\left(\rho_{\text {aire }}\right)+\left(\frac{\partial V_{20^{\circ} \mathrm{C}}}{\partial t_{\text {agua }}}\right)^{2} u^{2}\left(t_{\text {agua }}\right)+\left(\frac{\partial V_{20^{\circ} \mathrm{C}}}{\partial \alpha}\right)^{2} u^{2}(\alpha)+\left(\frac{\partial V_{20^{\circ} \mathrm{C}}}{\partial \rho_{\text {pesas }}}\right)^{2} u^{2}\left(\rho_{\text {pesas }}\right)
\end{array}\right]
$$




$$
\begin{aligned}
& u_{c}\left(V_{20^{\circ} \mathrm{C}}\right)=\sqrt{u_{c}^{2} \cdot\left(V_{20^{\circ} \mathrm{C}}\right)} \\
& \left(\frac{\partial V_{20^{\circ} \mathrm{C}}}{\partial M_{b+\text { agua }}}\right)^{2} u_{c}^{2}\left(M_{b+\text { agua }}\right)=\left(1,002786 \mathrm{~cm}^{3} / \mathrm{g}\right)^{2} \cdot(0,01530 \mathrm{~g})^{2}=2,35 \cdot 10^{-4} \mathrm{~cm}^{6} \\
& \left(\frac{\partial V_{20^{\circ} \mathrm{C}}}{\partial M_{b}}\right)^{2} u_{c}^{2}\left(M_{b}\right)=\left(-1,002786 \mathrm{~cm}^{3} / \mathrm{g}\right)^{2} \cdot(0,000072 \mathrm{~g})^{2}=5,21 \cdot 10^{-9} \mathrm{~cm}^{6} \\
& \left(\frac{\partial V_{20^{\circ} \mathrm{C}}}{\partial \rho_{\text {agua }}}\right)^{2} u_{c}^{2}\left(\rho_{\text {agua }}\right)=\left(-100,29610\left(\mathrm{~cm}^{3}\right)^{2} / \mathrm{g}\right)^{2} \cdot\left(1,0524 \cdot 10^{-5} \mathrm{~g} / \mathrm{cm}^{3}\right)^{2}=1,11 \cdot 10^{-6} \mathrm{~cm}^{6} \\
& \left(\frac{\partial V_{20^{\circ} \mathrm{C}}}{\partial \rho_{\text {aire }}}\right)^{2} u_{c}^{2}\left(\rho_{\text {aire }}\right)=\left(87,79397\left(\mathrm{~cm}^{3}\right)^{2} / \mathrm{g}\right)^{2} \cdot\left(1,56 \cdot 10^{-6} \mathrm{~g} / \mathrm{cm}^{3}\right)^{2}=1,88 \cdot 10^{-8} \mathrm{~cm}^{6} \\
& \left(\frac{\partial V_{20^{\circ} \mathrm{C}}}{\partial t_{\text {agua }}}\right)^{2} u^{2}\left(t_{\text {agua }}\right)=\left(-0,001 \mathrm{~cm}^{3} /{ }^{\circ} \mathrm{C}\right)^{2} \cdot\left(0,05{ }^{\circ} \mathrm{C}\right)^{2}=2,5 \cdot 10^{-9} \mathrm{~cm}^{6} \\
& \left(\frac{\partial V_{20^{\circ} \mathrm{C}}}{\partial \alpha}\right)^{2} u^{2}(\alpha)=\left(-37,002 \mathrm{~cm}^{3} \cdot{ }^{\circ} \mathrm{C}\right)^{2} \cdot\left(5,77 \cdot 10^{-6}{ }^{\circ} \mathrm{C}^{-1}\right)^{2}=4,56 \cdot 10^{-8} \mathrm{~cm}^{6} \\
& \left(\frac{\partial V_{20^{\circ} \mathrm{C}}}{\partial \rho_{\text {pesas }}}\right)^{2} u^{2}\left(\rho_{\text {pesas }}\right)=\left(0,001623\left(\mathrm{~cm}^{3}\right)^{2} / \mathrm{g}\right)^{2} \cdot\left(0,019999 \mathrm{~g} / \mathrm{cm}^{3}\right)^{2}=1,05 \cdot 10^{-9} \mathrm{~cm}^{6} \\
& u_{c}^{2}\left(V_{20^{\circ} \mathrm{C}}\right)=\left(2,35 \cdot 10^{-4}+5,21 \cdot 10^{-9}+1,11 \cdot 10^{-6}+1,88 \cdot 10^{-8}+2,5 \cdot 10^{-9}+4,56 \cdot 10^{-8}+1,05 \cdot 10^{-9}\right) \mathrm{cm}^{6} \\
& u_{c}^{2}\left(V_{20^{\circ} \mathrm{C}}\right)=2,365836 \cdot 10^{-4} \mathrm{~cm}^{6}
\end{aligned}
$$

Los valores numéricos se sustituyen en la ecuación (73).

$$
\begin{aligned}
& U_{V_{20^{\circ} \mathrm{C}}}= \pm 0,015381 \mathrm{~cm}^{3} \cdot 2 \\
& U_{V_{20^{\circ} \mathrm{C}}}= \pm 0,03 \mathrm{~cm}^{3}
\end{aligned}
$$

\subsubsection{Estimación del resultado de la medición}

El resultado de la medición pueden expresarse de una de las dos maneras siguientes:

a) Para el matraz de $100 \mathrm{~cm}^{3}$, analizado en el período del 30 al 31 de mayo del 2001, el volumen promedio a $20{ }^{\circ} \mathrm{C}\left(V_{20}{ }^{\circ} \mathrm{C}\right)$, medido a una temperatura promedio de $22,08{ }^{\circ} \mathrm{C}$ y una humedad relativa promedio del 53,7 \%, es de $100,004 \mathrm{~cm}^{3}$, con una incertidumbre estándar combinada de $0,015 \mathrm{~cm}^{3}$.

b) Para el matraz de $100 \mathrm{~cm}^{3}$, analizado en el período del 30 al 31 de mayo del 2001, el volumen promedio a $20{ }^{\circ} \mathrm{C}\left(V_{20}{ }^{\circ} \mathrm{C}\right)$, medido a una temperatura promedio de $22,08{ }^{\circ} \mathrm{C}$ y una humedad relativa promedio del $53,7 \%$, es de $(100,00 \pm 0,03) \mathrm{cm}^{3}$, donde la incertidumbre reportada es una incertidumbre expandida como lo define el Vocabulario Internacional de Metrología, primera edición en español del VIM-3 ${ }^{\mathrm{a}}$ del 2008, calculada usando un factor de cobertura, $\mathrm{k}=2$, que da una probabilidad de cobertura aproximada del $95 \%$.

\subsubsection{Error del matraz}

El error del matraz se calcula con la ecuación (74).

$$
\left(\frac{V_{\max }-V_{\min }}{V_{\max }}\right) \cdot 100=0,005 \%
$$




\subsubsection{Reproducibilidad en el tiempo}

La reproducibilidad en el tiempo se calcula con la ecuación (75).

$$
\overline{\mathrm{V}}_{20^{\circ} \mathrm{C}}-\mathrm{V}_{\text {nominal }}
$$

\section{CONCLUSIONES}

La experiencia obtenida en la realización de un ensayo intercomparación ha sido transmitida en este documento para que sirva como base en los procedimientos y métodos de cálculo de situaciones semejantes que se puedan presentar en laboratorios de calibración y ensayo, además ha sido para las autoras una experiencia interdisciplinaria de gran enriquecimiento.

\section{AGRADECIMIENTOS}

Al señor Pedro Antonio Rojas Camacho, técnico en metrología del Laboratorio de Productos Forestales del Instituto de Investigaciones en Ingeniería, de la Universidad de Costa Rica, por su apoyo en la consecución de los resultados analíticos y su gran interés por el desarrollo de la metrología en Costa Rica, así como al asistente Gabriel Ignacio Molina Castro, estudiante de Ingeniería Química de la Universidad de Costa Rica, por su apoyo en la comprobación de resultados matemáticos y estadísticos.

\section{REFERENCIAS BIBLIOGRÁFICAS}

ASTM (American Society for Testing Materials). (2009a). Standard specification for laboratory glass volumetric flasks. E 28806. In: Annual Book of ASTM Standards. Volume 14.04 Laboratory Apparatus; Degradation of Materials; SI; Oxygen Fire Safety. Philadelphia. 3 p.

ASTM (American Society for Testing Materials). (2009b). Standard practice for calibration of laboratory volumetric apparatus. E54201 (Reapproved 2007). In: Annual Book of ASTM Standards. Volume 14.04 Laboratory Apparatus; Degradation of Materials; SI; Oxygen Fire Safety. Philadelphia. 8 p.

ASTM (American Society for Testing Materials). (2009c). Standard specification for laboratory glass volumetric flasks, special use. E 1878-97 (Reapproved 2008). In: Annual Book of ASTM Standards. Volume 14.04 Laboratory Apparatus; Degradation of Materials; SI; Oxygen Fire Safety. Philadelphia. 2 p.

ASTM (American Society for Testing Materials). (2009d). Standard specification for laboratory glass volumetric apparatus. E 694-99 (Reapproved 2005). In: Annual Book of ASTM Standards. Volume 14.04 Laboratory Apparatus; Degradation of Materials; SI; Oxygen Fire Safety. Philadelphia. 7 p.

BIMP, IEC, IFCC, ILAC, ISO, IUPAP y OIML. Vocabulario Internacional de Metrología. Conceptos fundamentales y generales, y términos asociados (VIM). JCGM 200:2008. $1^{\mathrm{a}}$ Edición en español del VIM- $3^{\mathrm{a}}$, 2008. 88p.

Dávila, J. (2009). Necesidades industriales en la medición del contenido de humedad en gases. CENAM, México. 27 p. Extraído el 10 de febrero del 2010 de http://www.cenam. mx/DME/pdf/PRE_E-Mie_6.pdf

Dávila, J. \& Martines, E. (2006). Propagación de incertidumbre en la conversión de algunas magnitudes de humedad. Simposio de Metrología. 6 p.

Elert, G. (1998). Thermal expansion. Extraído el 05 de abril del 2010 de http://physics.info/ expansion/

ISO (International Organization for Standardization). (1978). International standard laboratory glassware. Principles of design and construction of volumetric 
glassware ISO 384. First edition, 1978.01.15.13p.

ISO (International Organization for Standardization). (1983). International standard laboratory glassware. One mark volumetric flasks ISO 1042. Third edition 1983.02.01. 5p.

ISO (International Organization for Standardization). (1984). International standard laboratory glassware. Volumetric glassware Methods for use and testing of capacity. ISO 4787. (1a ed.), 1984.11.15.15p.

Jones, F. \& Harris, G. (1992). ITS-90 Density of water formulation for volumetric standards calibration. Journal of Research of the National Institute of Standards and Technology, 97(3), 335-340.

OIML (Organización Internacional de Metrología Legal). (2004). Weights of classes E1, E2, F1, F2, M1, M1-2, M2, M2-3 and M3. Part 1: Metrological and technical requirements. OIML R 111-1. 80p.

Picard,A., Davis, R., Gläser, M. \& Fujii, K. (2008). Revised formula for the density of moist air (CIPM-2007). Metrologia, 45, 149-155.

Sánchez, M. E. (2001). Limpieza del matraz. Laboratorio Costarricense de Metrología (LACOMET), San José, 2p.

Sánchez, M. E. (2002). Calibración de equipo volumétrico: método gravimétrico, Laboratorio Costarricense de Metrología: LACOMET.

Sánchez, M. E.; Picado, C; Sánchez, H. \& Sequeira, M. Comparación interlaboratorios en Costa Rica ENA-MET-101. Calibración de un matraz de $100 \mathrm{~cm} 3$. Informe final. 2001.
Schimd, W. A. \& Martínez, R. (2004). Guía para estimar la incertidumbre de la medición. CENAM, México. 27 p.

Tanaka, M., Girard, G., Davis, R., Peuto, A. \& Bignell, N. (2001). Recommended table for the density of water between $0{ }^{\circ} \mathrm{C}$ and 40 ${ }^{\circ} \mathrm{C}$ based on recent experimental reports. Metrologia, 38, 301-309.

\section{REFERENCIAS BIBLIOGRÁFICAS RECOMENDADAS}

Becerra, L. O. \& Guardado, M. E. (2003). Estimación de la incertidumbre de la densidad del aire. CENAM, México. 23 p.

Trujillo, S. \& Arias, R. (2002). Incertidumbre en la calibración de un matraz volumétrico. CENAM, México. 18 p.

\section{SOBRE LAS AUTORAS}

\section{María Lorena Blanco Rojas}

Licenciada en Ingeniería Química de la Universidad de Costa Rica

M. Sc. en Ciencia y Tecnología de la Madera de la Universidad de São Paulo, Brasil

Profesora de la Escuela de Ingeniería Química Investigadora del Instituto de Investigaciones en Ingeniería

Universidad de Costa Rica

Teléfono: (506) 2511-6163

Correo electrónico: lorena@inii.ucr.ac.cr

\section{Paulina Silva Trejos}

Licenciada en Química de la Universidad de Costa Rica

Profesora de la Escuela de Química

Universidad de Costa Rica

Teléfono: (506) 2511-6163

Correo electrónico: stpaulinita@gmail.com 


\section{Análisis y comentarios}


